\title{
Prolonged QT Interval Associated with Multiple Entities
}

\author{
Alper $\mathrm{Alp}^{1^{*}}$, Ömer Faruk Geçin², İbrahim Altun ${ }^{3}$ \\ ${ }^{1}$ Muğla Sitkı Koçman University, School of Medicine, Nephrology, Muğla, Turkey \\ ${ }^{2}$ Muğla Sttkı Koçman University, School of Medicine, Internal Medicine, Muğla, Turkey \\ ${ }^{3}$ Muğla Sıtkı Koçman University, School of Medicine, Cardiology, Muğla, Turkey
}

Dear editor,

A 65-year-old female patient was admitted to the emergency department with the complaints of nausea, vomiting, and deterioration in general condition. Her medical history included hypertension, hyperlipidemia, and type 2 diabetes mellitus. At the same time, the patient had a mass of $14.9 \times 9 \mathrm{~cm}$ in the left ovary, which was detected about 2 weeks before, and investigations were ongoing (biopsy pending). The mass - potentially malignant extended up to the umbilicus and was compatible with colon invasion. Her medication included trimetazidine, sitagliptin/metformin, fenofibrate, gliclazide, and atenolol/ chlorthalidone. It was learned that the patient, who had a colonoscopic examination plan, had also been taking laxative agents for the last few days and was administered an enema containing phosphorus. Laboratory findings at admission are shown in Table 1. PCR for COVID-19 was negative. The patient's electrocardiography showed sinus rhythm, long corrected QT time (600 msec), and negative $\mathrm{T}$ waves in $\mathrm{V} 1-\mathrm{V} 3$. The two-dimensional echocardiogram showed normal left ventricular systolic function. Suspecting that the cause of QT prolongation in the patient was secondary to hypocalcemia, $30 \mathrm{~mL}$ of $10 \%$ calcium gluconate (225 mg of calcium gluconate monohydrate in each ampoule) were intravenously infused in 5\% dextrose fluid. A temporary jugular hemodialysis catheter was inserted, and hemodialysis was performed in the patient, who also had an emergency hemodialysis indication. Electrocardiograms taken the next day and the day after showed that the corrected QT distance returned to the normal range (480 msec and $230 \mathrm{msec}$, respectively).

Long QT syndrome presents with prolonged QT interval and T-wave abnormalities associated with delayed ventricular repolarization. Since QT prolongation can lead to Torsades de Pointes (QTC $>500$ ms increases this risk), it is a situation that needs to be analyzed seriously. ${ }^{1}$ The prolonged corrected QT time may be acquired or congenital in nature. Electrolyte disturbances and drugs are the most common causes of acquired cases; albeit less frequently, female gender, heart block, and hypothyroidism may also be contributing factors. ${ }^{2}$ Among these drugs, antiarrhythmics (e.g., quinidine, procainamide, disopyramide, sotalol, azimilide, dofetilide, amiodarone) are the leading group. Other classes of drugs (e.g., erythromycin, moxifloxacin, pentamidine, amantadine, chloroquine, trimethoprim-sulfamethoxazole, phenothiazines, astemizole, ketoconazole, ketanserin) may also present a similar effect. Hypokalemia, hypomagnesemia, and hypocalcemia are the most common electrolyte disturbances that lead to QT prolongation. Our patient had hypocalcemia and hypokalemia at admission. However, the next day, while the QT distance returned to normal with calcium replacement, hypokalemia was still persisting. This indicated that the QT prolongation effect may primarily be secondary to hypocalcemia. Although the phosphorus level of the patient was 
TABLE 1. Laboratory findings

\begin{tabular}{lcc}
\hline Parameter & On admission & $\begin{array}{c}\text { Next day } \\
\text { (after first } \\
\text { hemodialysis) }\end{array}$ \\
\hline Urea (mg/dL) & 323.1 & 218.5 \\
Creatinine (mg/dL) & 8.93 & 5.23 \\
GFR (ml/min/1.73 m²) & 4 & \\
Sodium (136-145 mmol/L) & 131 & 132 \\
Potassium (3.5-5.1 mmol/L) & 3.33 & 3.2 \\
pH & 7.53 & 7.46 \\
HCO & 59 & 28.4 \\
LDH (135-214 U/L) & 554 & 309 \\
Albumin (3.5-5.2 g/dL) & 3.96 & 3 \\
Magnesium (1.5-2.5 mg/dL) & 2.85 & 2.1 \\
Hemoglobin (g/dL) & 10.1 & 8.2 \\
Hematocrit (\%) & 30.8 & 25.2 \\
Calcium (8.6-10 mg/dL) & 6.76 & 9.17 \\
Phosphorus (2.5-4.5 mg/dL) & 11.69 & 8.2 \\
Uric acid (2.4-5.7 mg/dL) & 22.4 & 6.3 \\
Parathormone (pg/mL) & 319 & $\mathrm{n} / \mathrm{a}$ \\
\hline
\end{tabular}

very high, calcium replacement was performed together with hemodialysis due to the possibility of malignant arrhythmia. Although the prolongation of QTc interval is proportional to the level of hypocalcemia, interestingly, the calcium level in our patient was closer to normal levels than in similar cases in the literature. ${ }^{3}$ Concomitant hypokalemia and severe kidney damage may have influenced QT prolongation. In conclusion, hypocalcemia, hypokalemia, possible ovarian cancer, and acute kidney damage may have had synergistic effects on QT prolongation in this patient. With effective calcium replacement, the QT interval has reached normal levels. Possible contrast nephropathy in the patient may have worsened this clinical condition. In addition, although histological diagnosis and hyperkaliemia were not accompanied, prerenal acute kidney damage due to colon cleansing and spontaneous tumor lysis syndrome may have contributed to the existing renal involvement, when taking into consideration the tumor size. As a result, acute kidney injury with multiple etiologies was considered primarily in the etiology of hypocalcemia-associated long QTc in this case. The patient agreed with the publication of her case, without images of parts of her body that could confirm her identity. The Ethics Committee of the Rehabilitation Hospital approved the publication of this report.

\section{CONFLICT OF INTEREST}

Nothing to declare.

\section{REFERENCES}

1. Gupta P, Agstam S, Yadav A, Ghosh S. Malignant prolongation of the QTC interval due to severe vitamin D deficiency: an unusual presentation. BMJ Case Rep. 2020;13:e237157. doi: 10.1136/bcr-2020-237157.

2. Rentoukas E, Lazaros G, Sotiriou S, et al. Extreme but not life-threatening QT interval prolongation? Take a closer look at the neck! J Electrocardiol. 2013;46:128-130. doi: 10.1016/j. jelectrocard.2012.10.007.

3. Gardner JD, Calkins JB Jr, Garrison GE. ECG diagnosis: The effect of ionized serum calcium levels on electrocardiogram. Perm J. 2014;18:e119-e120. doi: 10.7812/TPP/13-025. 